\title{
Coding for Minimizing Energy in VLSI Interconnects
}

\author{
K. S. Sainarayanan, J. V. R. Ravindra, Student Member, IEEE, Kiran T. Nath Student Member, IEEE, and \\ M. B. Srinivas, Member, IEEE
}

\begin{abstract}
In CMOS VLSI circuits, the dynamic power dissipation contributes a significant fraction in the overall power dissipation. Hence, the main target of VLSI designers is to minimize the switching activity on the on-chip bus lines. In this paper, the authors propose a novel bus encoding technique which minimizes both self and coupling transition activity to curtail the global power dissipation. The performance of the proposed coding scheme has been tested on various SPEC'95 benchmarks and found that with respect to unencoded data, an average reduction of $28 \%$ and $26 \%$ with respect to self and coupling energies in the total power dissipation is achieved. The hardware, used for encoding and decoding purposes, has been designed using Magma(c)tools.
\end{abstract}

Index Terms-Low power, Interconnect, Crosstalk, VLSI

\section{INTRODUCTION}

$\mathbf{M}$ OORE'S law says that the performance of an IC doubles every 18 months, the interconnect performance is expected to double only over the next decade [1]. This disparity, caused due to the various inductive and capacitive effects, poses a major challenge to VLSI system designers. Much of the power in a bus is dissipated in the process of charging and discharging the high nodal and inter-wire capacitances. Hence, majority of the works [2, 3, 4, 5], which exist in the literature focus on minimizing the bus transition activity. A very popular method among them is the Bus Invert method [3], which does a conditional inversion of the bus lines to minimize the self transitions and thereby reducing the self energy. As we approach Deep Submicron (DSM) and Ultra Deep Submicron (UDSM) technologies, the effect of interwire capacitance becomes significant due the high proximity of the bus lines carrying signals. As a result, many attempts [4-9] to reduce the coupling transition activity also exists. In this paper, the authors propose a new coding technique which minimizes both coupling and self transition activities in the bus lines.

The rest of the paper is organized as follows: Definitions of some of the important terms which are used in this paper are given in section II. The problem formulation is given in section III and a brief overview of the previous works related to bus coding is given in section IV. The energy model is shown in section V. The proposed coding scheme is explained in section VI and its hardware implementation is shown in section

K. S. Sainarayanan, J. V. R. Ravindra, Kiran T. Nath, M. B. Srinivas are with Center for VLSI and Embedded System Technologies (CVEST), International Institute of Information Technology (IIIT), Gachibowli, Hyderabad 500032, Andhra Pradesh, India. Email:\{kssai,ravindra $@ @$ research.iiit.ac.in kirantnath@students.iiit.ac.in, srinivas@iiit.ac.in
VII. While, illustration of the proposed coding scheme with an example is shown is section VIII, the results and discussions are provided in section IX. Finally, conclusions are made in section $\mathrm{X}$.

\section{DEFINITIONS}

1) Coupling Transition (CT): A Coupling Transition is defined as a transition from $0 \rightarrow 1$ or $1 \rightarrow 0$, between two adjacent bus wires.

2) Self Transition (ST): A Self Transition is defined as a transition from $0 \rightarrow 1$ or $1 \rightarrow 0$ on bus with reference to the previous data on it.

3) Bus Width $(B W)$ : The number of bits in the data is called the Bus Width.

\section{PROBLEM Formulation}

In general, four sources of power dissipation can be identified in any CMOS VLSI circuit and they are related to the global power dissipation [6] by (1).

$$
P_{\text {Dissipation }}=P_{\text {Static }}+P_{\text {Dynamic }}+P_{\text {Leakage }}+P_{\text {Shortckt }}
$$

The major share in the overall power dissipation is that of dynamic power dissipation. Hence, this paper concentrates on reducing $P_{\text {Dynamic }}$ using a novel encoding technique described in section VI. Furthermore, the dynamic power dissipation in a CMOS VLSI circuit is given by

$$
P_{\text {Dynamic }}=\alpha * V_{D D}^{2} * C_{L} * f
$$

where, $\alpha$ is the transition activity factor, $V_{D D}$ is the supply voltage, $C_{L}$ is the load capacitance and $f$ is the frequency of operation. It is clear from (2) that, in order to minimize the dynamic power dissipation, attempts must be made to reduce one or more factors on the right hand side. In this paper, the authors focus on lowering the activity factor, which is a promising way of reducing the power dissipation. The transition activity factor $\alpha$ is given by

$$
\alpha=\alpha_{s} C_{s}+\alpha_{c} C_{c}
$$

where, $\alpha_{s}$ is the self transition activity factor which arises from the changes in a particular bus line, $C_{s}$ is the self capacitance i.e. the capacitance between the bus wire and the ground, $\alpha_{c}$ is the coupling transition activity factor which arises due to the difference in bits carried by adjacent bus lines and $C_{s}$ is the coupling capacitance i.e. the capacitance between two adjacent bus wires. 


\section{Previous Work}

The bus coding techniques existing in the literature, which attempt to reduce the power dissipation, can be classified into two major categories.

One set of methods aim at reducing the transitions in the address busses. Some of them are $A^{2} B C$ [11], The Beach Solution [12] and Gray Coding [13]. These techniques exploit the redundancy to minimize the power dissipation.

The other set of methods aim at reducing the transitions in the data buses. Among them, Bus Invert [3] is an efficient method for reducing the power dissipation by minimizing the self transitions. According to this method, if the number of bits that change is more than half the width of the bus, then the entire bus is inverted and transmitted. For decoding purposes, an extra bit is transmitted with the data which shows if the data in the bus is inverted or not. The main disadvantage of this method is that the authors concentrated only on self transitions. For DSM technology, the coupling effects become more dominant and Samala et al. proposed [4], which aims at reducing the coupling capacitance. In this method, the power consumed by their huge circuit would surpass the power savings and hence is not suitable for low power applications. Some of the other works existing in this field are Shift Invert [8] method and Coupling Driven Bus Coding [15]. A majority of the encoding techniques currently existing in literature do not consider both the self and coupling effects, while this paper considers the effects of both self and coupling transitional activities to minimize the power dissipation.

\section{ENERGY MODEL}

The DSM model of $n$ interconnects in parallel, driven by an inverter [2] is shown in Fig. 1. Here, $C_{s}$ and $C_{c}$ are the self and coupling capacitances, $R$ represents the on-off resistance of the driver, $V_{j}^{i}$ and $V_{j}^{f}$ denote the initial and final voltages in the $j^{\text {th }}$ interconnect.

The equation for calculating the total energy dissipation is given by (4) [2]. Equation (5) and Equation (6) describe the energy dissipation due to self transition, $E_{r}^{L}$, and coupling transitions, $E_{j}^{I}$, respectively. The subscript represents the node number and the superscript represents the initial or final value of voltage on the interconnect at that node. For example, $V_{j+1}^{f}$ represents the final value of voltage at $(j+1)^{t h}$ wire. $\alpha$ in (4) represents the technology parameter which is given by (7). The significance of $\alpha$ is that it's value becomes high when the technology shrinks.

$$
E=\sum_{r=1}^{N} E_{r}^{L}+\alpha \sum_{r=1}^{N-1} E_{r}^{I}
$$

where,

$$
\begin{gathered}
E_{r}^{L}=C_{s} V_{r}^{f}\left(V_{r}^{f}-V_{r}^{i}\right) \\
E_{j}^{I}=C_{c}\left(V_{j+1}^{f}-V_{j}^{f}\right)^{2}+\left(V_{j+1}^{f}-V_{j}^{f}\right)\left(V_{j+1}^{i}-V_{j}^{i}\right) \\
\alpha=\frac{C_{c}}{C_{s}}
\end{gathered}
$$

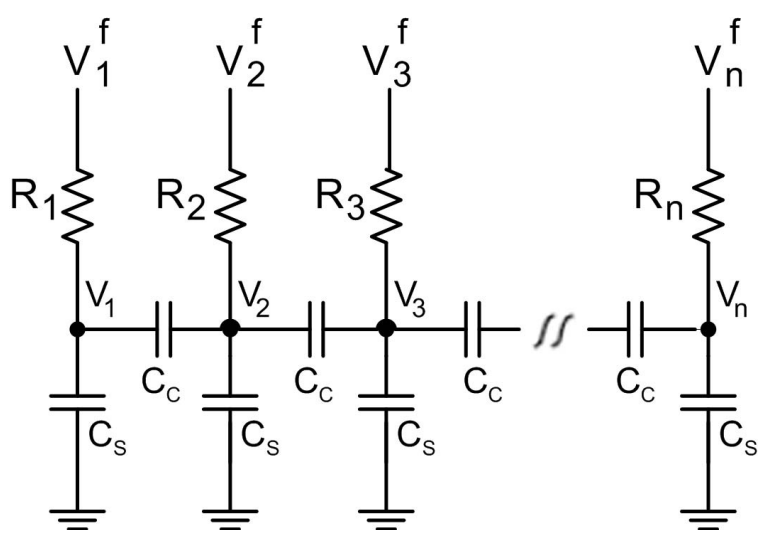

Fig. 1. Deep Submicron Model of N Interconnects in Parallel, Driven by an Inverter

\section{Proposed Coding Scheme}

Let the data on an $n$ bit wide bus, at time instant $t$ be denoted as $A^{t}=\left\{a_{n-1}^{t}, a_{n-2}^{t}, \ldots \ldots . a_{1}^{t}, a_{0}^{t}\right\}$. The data transmitted on the bus is denoted as $A^{(t) e n c}$. The function calculateST_n(data1,data2) finds the number of self transitions between (data1, data2). Here, data1 and data2 should be $n$ bits wide. The function swapAdj_n $\left(A^{t}\right)$ swaps the adjacent bits in $A_{t}$ and gives the output $A^{s w(t)}$ $=\left\{a_{n-1}^{t}, a_{n-2}^{t}, \ldots \ldots . a_{0}^{t}, a_{1}^{t}\right\}$.

The proposed coding scheme is as follows:

1) Let $A^{(t-1) e n c}$ be the previously coded data which was transmitted on the bus and let $A^{t}$ be the present data which should be encoded and transmitted.

2) Invert the odd lines in $A^{t}$ and affix it with 00 , for decoding puposes. Let this new data be denoted as $A^{t(o d d)}$

Evalute $\boldsymbol{s t} \_$odd $=$calculateST_n( $\left(A^{t(o d d)}, A^{(t-1) e n c}\right)$.

3) Likewise, Invert the even lines in $A^{t}$ and affix it with 01 . Let this new data be denoted as $A^{t(e v e)}$ Evalute st_eve=calculateST_n( $\left.A^{t(e v e)}, A^{(t-1) e n c}\right)$.

4) Let $A^{s w(t)}=\mathbf{s w a p A d j} \mathbf{n}\left(A^{t}\right)$. Suffix $A^{s w(t)}$ with 10 and let this new data be denoted as $A^{t(s w p)}$. Evalute $\boldsymbol{s t} \_\boldsymbol{s} \boldsymbol{w} \boldsymbol{p}=\mathbf{c a l c u l a t e S T} \mathbf{n}\left(A^{t(s w p)}, A^{(t-1) e n c}\right)$.

5) Suffix $A^{t}$ with 11 and let this new data be denoted as $A^{t(u n c)}$.

Evalute $\boldsymbol{s t}_{\text {_unc}}=\mathbf{c}$ calculateST_n$\left(A^{t(u n c)}, A^{(t-1) e n c}\right)$.

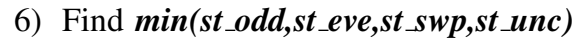

7) The coded pattern corresponding the minimum value in step 6 is transmitted.

\section{HARDWARE IMPLEMENTATION}

The block diagram of the proposed encoder is given in Fig. 2. The encoder takes an $n$ bit input. The calculateST_n functions in the steps $\mathbf{2}, \mathbf{3}, \mathbf{4}, \mathbf{5}$ in the coding scheme above are evaluated by the Energy Estimator block. The outputs of the Energy Estimator block, $\min \left(s t_{-} o d d, s t \_e v e, s t_{\text {swp }}, \boldsymbol{s t} \_\right.$unc) are compared among themselves and the minimum amongst them is found out. The encoded data pattern corresponding to the minimum value of self transitions is transmitted on the 


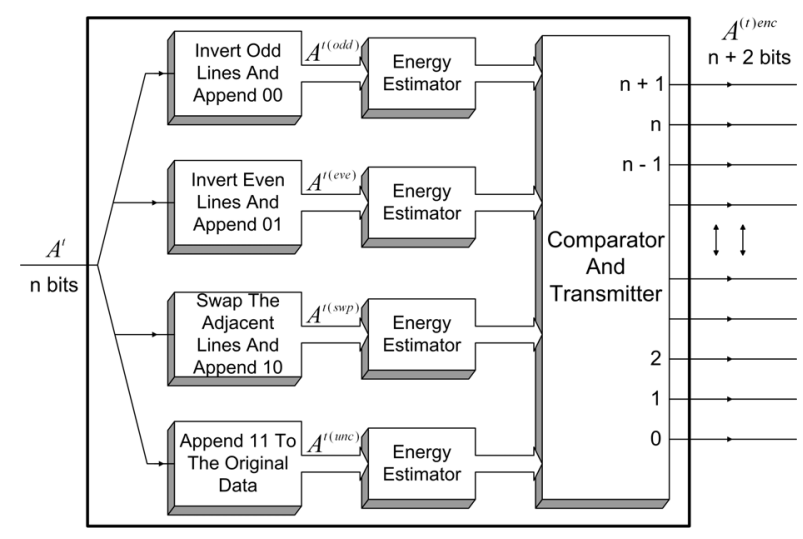

Fig. 2. Block Diagram of the Proposed Encoder

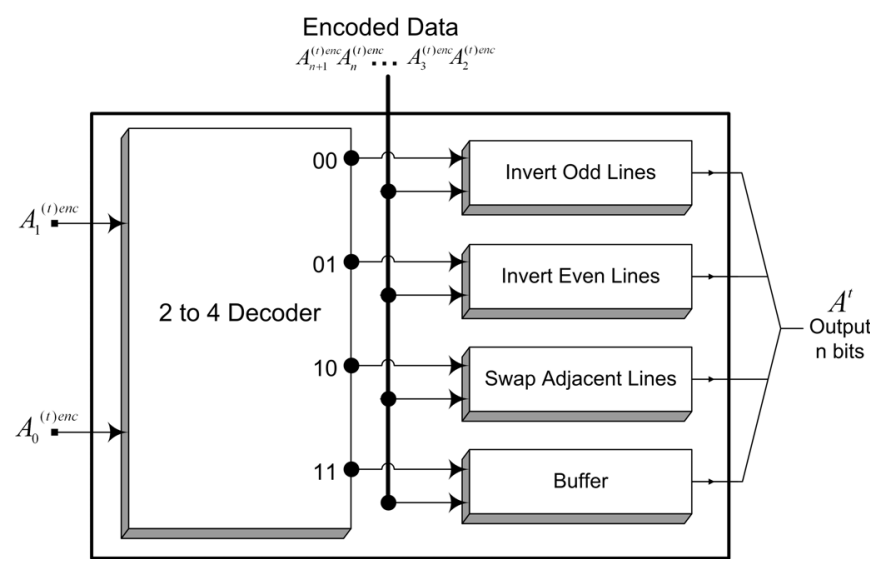

Fig. 3. Block Diagram of the Proposed Decoder

bus.

The decoder to be used at the receiving end is shown in Fig. 3. The two least significant bits in the transmitted pattern are given to a 2 to 4 decoder and depending on these two bits, the appropriate decoding procedure is done.

The internal diagram of a 10 bit of Energy Estimator block is shown in Fig. 4. One of the input binary vectors is $A^{(t-1) e n c}$, which is the previous data on the bus at time (t-1) and other input is $\left\{A^{(t) e n c i}\right\} \in$ $\left\{A^{t(\text { odd })}, A^{t(\text { eve })}, A^{t(s w p)}, A^{t(u n c)}\right\}$ in sequence. The array of the output binary vectors is $\left\{B_{3} B_{2} B_{1} B_{0}\right\}$. We have used the concept of parallel adders in order to minimize the critical delay. The array of EXOR gates is shown in Fig. 4 performs the action of a coincidence circuit. In this particular example, it can be seen that for a '10' bit Energy Estimation, we get a 4 bit output. In general, for a $w$ bit wide connector, the output of the Energy Estimator will be $\left\lceil\log _{2}(w)\right\rceil$ bit wide.

\section{Illustation of the Proposed Coding Scheme}

Let the coded data on the bus at time (k-1) be $A^{(k-1) e n c}=\{\uparrow \downarrow \uparrow \downarrow \downarrow \downarrow \downarrow \uparrow \downarrow \uparrow\}$ and let $A^{k}=\{\uparrow \uparrow \uparrow \uparrow \downarrow \uparrow \downarrow \uparrow\}$ be the current data that should be transmitted after encoding. In this case number of transitions without coding is 7 and with coding is given by,

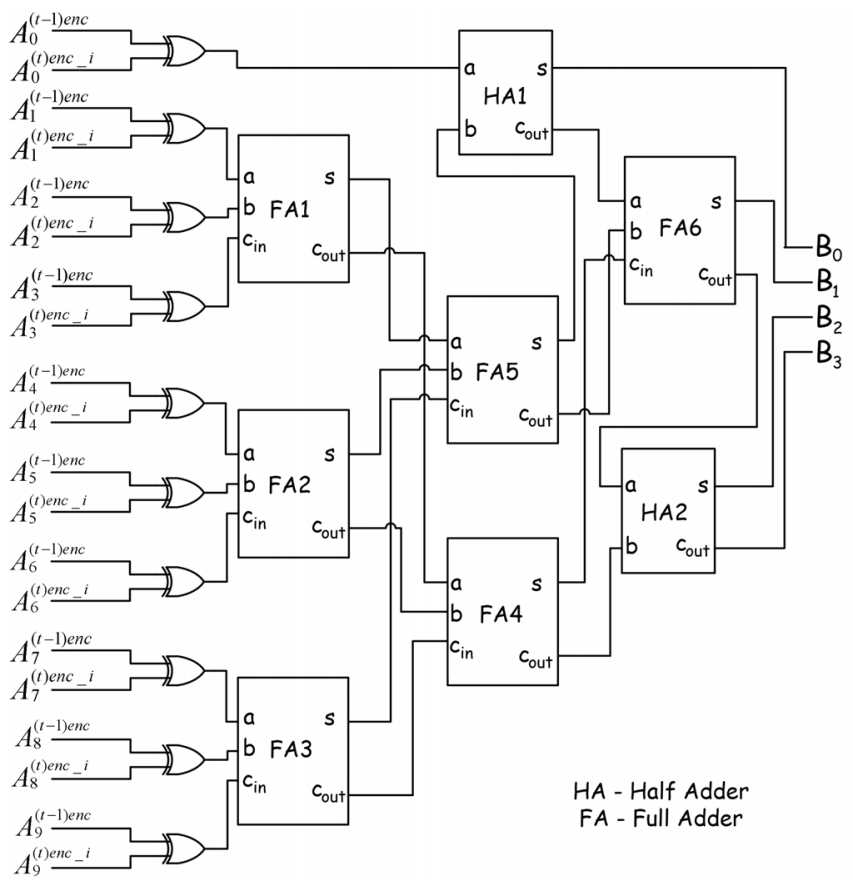

Fig. 4. Hardware Implementation of the Energy Estimator Block

$$
\begin{aligned}
A^{t(o d d)} & =\{\uparrow \downarrow \uparrow \downarrow \downarrow \downarrow \downarrow \downarrow \downarrow \downarrow\} \\
A^{t(e v e)} & =\{\downarrow \uparrow \downarrow \uparrow \uparrow \uparrow \uparrow \uparrow \downarrow \uparrow\} \text { with st_odd=2 } \\
A^{t(s w p)} & =\{\uparrow \uparrow \uparrow \uparrow \uparrow \uparrow \downarrow \uparrow \downarrow \uparrow \downarrow\} \text { st_eve }=7 \\
A^{t(u n c)} & =\{\uparrow \uparrow \uparrow \uparrow \uparrow \downarrow \uparrow \downarrow \uparrow \uparrow \uparrow \uparrow\} \text { with st_swp=7 }
\end{aligned}
$$

Since $\min \left(s t_{-}\right.$odd,st_eve,st_swp,st_unc$)=($st_odd $), A^{t(o d d)}$ is transmitted. Hence $A^{(t) e n c}=A^{t(o d d)}$ and the number of transitions is reduced from $\mathbf{7}$ to $\mathbf{2}$ with respect to the transmission of uncoded data.

\section{Simulation Results and Discussions}

The power dissipation of various coding schemes has been tested with 1,00,000 random vectors for 8 and 16 bit wide bus and the observations are tabulated in Table I. Partitioning of bus lines for buses of higher width $(32,64,128$ bit widths etc.) will give a better power saving than applying the coding directly for the entire bus width [15]. Partitioning of bus lines have not been considered in this paper. The value of the technology parameter, $\alpha$, increases as the technology shrinks. Experiments have been conducted for various values of the technology parameter and the results are shown in Table I where, $\alpha=0$ signifies the bus model without coupling effects, $\alpha=3.5$ represents $130 \mathrm{~nm}$ technology and $\alpha=5$ represents $90 \mathrm{~nm}$ technology. It can be inferred from Table I that the proposed method is efficient when compared to various methods proposed earlier in the literature in terms of reduction in power dissipation with respect to both self and coupling energies. The performance of the proposed coding scheme has been evaluated using SPEC'95 benchmark suite [16] considering the extra bits added for decoding purpose. The results obtained are shown in Table II. In general, any coding technique should consider power dissipation due to coding/decoding hardware (CODEC) also in order to estimate true power saved. Thus, the 
TABLE I

PERFORMANCE OF THE PROPOSED CODING TECHNIQUE WITH OTHER CODING TECHNIQUES (EXPRESSED AS PERCENTAGE), CONSIDERING THE EXTRA BITS ADDED THE ENCODER

\begin{tabular}{|c||c|c|c||c|c|c|}
\hline \multirow{2}{*}{\multicolumn{1}{|c||}{ BCT }} & \multicolumn{3}{c|}{ BW=8 bit } & \multicolumn{3}{c|}{ BW=16 bit } \\
\cline { 2 - 7 } & $\alpha=0$ & $\alpha=3.5$ & $\alpha=5.0$ & $\alpha=0$ & $\alpha=3.5$ & $\alpha=5.0$ \\
\hline BI[3] & 15.9 & 14.2 & 14.0 & 13.6 & 13.5 & 13.5 \\
\hline NBED[4] & 9.5 & 8.98 & 9.31 & 8.5 & 9.0 & 8.32 \\
\hline DSM BI[9] & 19.1 & 18.82 & 17.32 & 16.14 & 16.0 & 16.1 \\
\hline SHIV[7] & 20.6 & 18.6 & 18.5 & 17.1 & 18.0 & 18.0 \\
\hline OE-BI[6] & 23.1 & 22.45 & 23.0 & 20.12 & 19.98 & 20.02 \\
\hline GC[17] & 22.0 & 21.32 & 22.0 & 19 & 18.12 & 18 \\
\hline DC[13] & 17.32 & 18.12 & 17.14 & 16.4 & 16.6 & 16.1 \\
\hline NBC[8] & 22.65 & 21.21 & 20.0 & 21.62 & 21.0 & 25.9 \\
\hline PM $^{1}$ & 28.32 & 27.14 & 27.0 & 26.32 & 26.5 & 25.9 \\
\hline
\end{tabular}

TABLE II

PERFORMANCE OF THE PROPOSED CODING TECHNIQUE ON SPEC'95 BENCHMARKS CONSIDERING THE TWO EXTRA BITS ADDED BY THE ENCODER

\begin{tabular}{|c||c|c|}
\hline \multicolumn{1}{|c||}{ Benchmark } & \multicolumn{2}{c|}{ Power Saved } \\
\cline { 2 - 3 } & Coupling Energy & Self Energy \\
\hline compress & 26.5 & 25.9 \\
\hline go & 24.12 & 22.9 \\
\hline gcc & 28.10 & 25.45 \\
\hline vortex & 25.12 & 24.38 \\
\hline mpeg & 22.9 & 22.3 \\
\hline
\end{tabular}

actual total power saved, $P_{\text {total }}$ is given by (8), where $P_{s}$ is the power saved due to encoding technique and $P_{\text {codec }}$ is the power dissipated by the CODEC relative to the un-encoded bus.

$$
P_{\text {total }}=P_{s}-P_{\text {codec }}
$$

Since, power dissipation due to CODEC has not been considered by earlier coding techniques, CODECs have been designed by the authors for a few select coding techniques and the total power saved has been estimated using (8). The results are illustrated in Table III. It can be seen that the total power saved by the proposed method is much higher than the other methods. Low energy in NBED [4] method signifies that the power dissipated by the CODEC surpasses the power saved by the coding scheme.

TABLE III

THE TOTAL POWER SAVED OF VARIOUS CODING SCHEMES (EXPRESSED AS PERCENTAGE)

\begin{tabular}{|c|c|}
\hline Bus Coding Technique & \% Total Power Saved \\
\hline BI[3] & 13.2 \\
\hline NBED[4] & 2.52 \\
\hline DSM BI[9] & 14.5 \\
\hline SHIV[7] & 15.5 \\
\hline OE-BI[6] & 18.8 \\
\hline Generic Coding[16] & 18.2 \\
\hline Dynamic Coding [13] & 14.12 \\
\hline NBC[8] & 16.55 \\
\hline Proposed Method & 23.01 \\
\hline
\end{tabular}

\section{COnClusions}

A new bus coding technique, which reduces the power dissipation with respect to both coupling and self energies, has been proposed. This technique achieves a reduction of $28 \%$ and $26 \%$ in self and coupling energies respectively. Experimental results have shown that the proposed method is suitable for the continually shrinking technology. The CODEC for the proposed coding scheme has been designed using Magma (c)tools. The total power saved considering the power overhead of the CODEC is found to be $23 \%$. Hence the proposed coding scheme is suitable for low power VLSI applications.

\section{ACKNOWLEDGEMENT}

The Authors would like to thank Magma(C)Design Automation (India) pvt. Ltd, Bangalore for sponsoring Magma(c)tools used in this work.

\section{REFERENCES}

[1] International Technology Roadmap for Semiconductors. http://public.itrs.net

[2] P. Sotiriadis and A. Chandrakasan, "Low power Coding Techniques Considering Inter wire capacitances," In Proc. Of IEEE Conferences on Custom Integrated Circuits (CICC'O0), pp 507-510, 2000

[3] M.R Stan and W.P Burelson, "Bus Invert Coding for Low Power I/O," IEEE Transactions VLSI systems, pp 49-58, March 1995.

[4] N.K. Samala, R. Damu and B.Izadi "Novel Deep Submicron Bus Coding for Low Energy," In Proceedings of the Embedded System and Applications, pp. 25-30 June 2004.

[5] P. Sotiriadis, "Interconnect Modeling and Optimization in Deep Submicron Technologies," Dissertation Thesis, Massachusetts Institute of Technology, May 2002.

[6] Yan Zhang et al, "Odd/Even bus invert with two phase transfer for buses with coupling," Proceedings of ISPLED '02, pp 80-83, August 12-14, Monterey, CA, USA.

[7] Jayapreetha Natesan and Damu Radhakrishnan, "Shift Invert coding (SINV) for low power VLSI," Proceedings of EUROMICRO Systems on Digital System Design (DSD'04), pp 190-194, 2004.

[8] J.V.R Ravindra, K.S. Sainarayanan, M.B. Srinivas, "A novel bus coding technique for low power data transmission," IEEE symposium on VLSI design and test conference (VDAT-2005), pp 263-266, August 2005.

[9] Lindkvist, T., Lofvenberg, J.; Gustafsson, O., "Deep sub-micron bus invert coding," Proceedings of the 6th Nordic Signal Processing Symposium, pp 133-136, 2004

[10] Jorg Henkel, Haris Lekatsas, “ $A^{2} B C$ : Adaptive Address Bus Coding for Low Power Deep SubMicron Designs," Proceedings of DAC, pp. 744 $-749,2001$.

[11] Benini, L et. al, "System-Level Power Optimization of Special Purpose Applications: The Beach Solution," Proceedings of International Symposium on Low Power Electronics and Design, pp 24-29, Aug 1997.

[12] Su, C.L et al, "Saving power in the control path of embedded processors," Design \& Test of Computers, IEEE, pp 24-31, 1994.

[13] Madhu, M. et al, "Dynamic coding technique for low-power data bus," Proceedings of IEEE Computer Society Annual Symposium on VLSI, pp 252-253, 2003.

[14] Ki-Wook Kim et al, "Coupling-driven signal encoding scheme for low- power interface design," Proceedings of IEEE/ACM International Conference on Computer Aided Design(ICCAD), pp 318-321, 2000.

[15] Lin Xie et al, "Partitioned Bus Coding for Energy Reduction," Proceedings of ASPDAC 2005, pp1280-1283, 2005

[16] Spec 95 benchmark suite: http://spec.com

[17] Zahid Khan et al "Novel Bus Encoding Scheme from Energy and Crosstalk Efficiency Perspective for AMBA based Generic SoC Systems," Proceedings of VLSID , pp 751-756, 2005. 